\title{
Estudios citogenéticos de dos especies y una variedad del género Nyctocereus (Cactaceae)
}

\author{
Guadalupe Palomino Hasbach, Socorro Zuleta Lechuga y Leia \\ SCHEINVAR ${ }^{1}$
}

RESUMEN. Nyctocereus (Cactaceae) es un género con siete especies y dos variedades distribuidas de México a Centroamérica, todas son ornamentales y sus frutos son comestibles. En este estudio se determinaron los números cromosómicos y se elaboraron los cariotipos de $N$. castellanosii, $N$. serpentinus y $N$. serpentinus var. splendens. El número cromosómico para los tres taxa fue $2 n=22$ y el número básico $\mathrm{x}=11$. Entre ellos el cariotipo es muy homogéneo. No se observaron poliploidías. Todos los cromosomas en $N$. castellanosii y $N$. serpentinus fueron metacéntricos. En $N$. serpentinus var. splendens se obtuvieron dos partes de submetacéntricos. La longitud total de la cromatina fue diferente en las tres especies, presentando la menor longitud $N$. serpentinus var. splendens.

ABSTRACT. The genus Nyctocereus (Cactaceae) consists of seven species and two varieties distributed from Mexico to Central America. They are valued as ornamental and for its edible fruits. In this study the chromosome numbers were determined and the karyotypes elaborated for $N$. castellanossi, $N$. serpentinus and $N$. serpentinus var. splendens. The diploid chromosome number for the three taxa was $2 n=22$ and the karyotypes were very homogenous. No polyploids were detected. The basic number is $\mathrm{x}=11$. All the chromosomes in $N$. castellanosii and $N$. serpentinus were metacentric, while in $N$. serpentinus var. splendens two pairs of submetacentric chromosomes were observed. The total chromatin lenght was different for the three species, finding the lowest value in $N$. serpentinus var. splendens.

El género Nyctocereus (Berg.) Britt. y Rose pertenece a la familia de las cactáceas. Son plantas terrestres o rupícolas y crecen en pedregales de origen volcánico. Sus especies se encuentran distribuidas desde México hasta Centroamérica; son ornamentales, con flores grandes, perfumadas, y sus frutos, llamados "pitayas", son comestibles (Bravo, 1978; Scheinvar, 1982).

Hay pocos trabajos citogenéticos en el género: Beard (1937) reportó un $n=11$ para

'Jardín Botánico, Instituto de Biología, Universidad Nacional Autónoma de México, Apdo. Postal 70-233, 04510, México, D. F.

Palomino-Hasbach G, Zuleta-Lechuga S, Scheinvar L. 1988. Estudios citogenéticos de dos especies y una variedad del género Nyctocereus (Cactaceae). Boletín de la Sociedad Botánica de México 48: 75-80. 
$N$ serpentinus en la metafase 1 de la meiosis, dato congruente con el número básico (x): $\mathrm{x}=11$, reportado para muchas otras cactáceas (Beard, 1937; Remski, 1954; Spencer, 1955; Pinkava y McLeod, 1971; Hiroshi et al., 1976; Johnson, 1978; Weedin y Powell, 1978). La poliploidía es un proceso común reportado para algunas cactáceas como el género Mammillaria donde se han reportado desde variedades diploides como M. prolifera var. arachnoidea (Johnson, 1978 y 1980) hasta 24 ploides como en M. capensis var. palida (Remski 1954) y en Opuntia spp, Hiroshi et al. (1976), refieren hasta 20 ploides.

$\mathrm{Al}$ parecer las poliploidías se dan como una adaptación a condiciones climáticas extremas como el frío (Stockwell, 1935; Johnson, 1980) aunque Hiroshi et al. (1976) afirman que ésto no siempre es aplicable a cactáceas.

La morfología de los cromosomas en representantes de las cactáceas tiene pocas variaciones (Remski, 1954; Pinkava y Mc Leod, 1971) presentándose en su mayoría de un tamaño uniforme y tendiendo a metacéntricos (Johnson, 1980).

En el género Mammillaria han sido reportados satélites en especies diploides y hexaploides (Johnson, 1980), así como aneuploidías en el género Echinocereus (Beard, 1937) e irregularidades meióticas fueron señaladas en algunas especies de los géneros Opuntia y Echinocereus (Weedin y Powell, 1978), y en Mammillaria, Johnson (1980).

Green et al. (1980) señalan que el análisis del cariotipo puede ser usado como instrumento en citotaxonomía o filogenética. Consideran que este recurso puede ayudar a la elaboración de mejores clasificaciones taxonómicas, así como a un mejor entendimiento de las relaciones evolutivas de los distintos niveles taxonómicos de los individuos.

En el presente trabajo se obtuvo el número y la forma de los cromosomas de Nyctocereus castellanosii Scheinvar, $N$, serpentinus (Lag. y Rod.) Britt. y Rose y N. serpentinus (Lag. y Rod.) Britt. y Rose var. splendens (DC) Berg. para analizar las diferencias y semejanzas en relación con la fórmula cariotípica, longitud de la cromatina e índice de asimetría en los cariotipos y proporcionar apoyo a los estudios taxonómicos y biosistemáticos que se llevan a cabo en el género.

\section{MATERIAL y MÉTODOS}

Las plantas utilizadas en este estudio pertenecen a la colección del Jardín Botánico de la UNAM. Se presentan los datos de las localidades donde fueron colectadas y los números de los ejemplares de herbario (MEXU) que los respaldan (tabla 1).

Las semillas fueron colectadas de las mismas plantas y germinadas en cajas de Petri esterilizadas conteniendo algodón y papel filtro humedecidos con agua destilada, manteniéndolas a $30^{\circ} \mathrm{C} \pm 1{ }^{\circ} \mathrm{C}$. Cuando las plantas tenían $2-3 \mathrm{~cm}$ de longitud, fueron transferidas a macetas con tierra de hoja. También se enraizaron fragmentos de tallos en arena de lava volcánica esterilizada y humedecida con la solución nutritiva de Hoagland. Cuando se obtenían las raíces secundarias de las plántulas y de los framgentos de tallos y alcanzaban de 2 a $3 \mathrm{~cm}$ de longitud, fueron pretratadas en una solución saturada de $\alpha$ bromonaftaleno por 2.5 horas a $18{ }^{\circ} \mathrm{C}$ y fijadas en una solución de alcohol etílico absoluto-ácido acético (3:1) durante 24 horas e hidrolizadas en $\mathrm{HC} 11 \mathrm{~N}$ a $60^{\circ} \mathrm{C}$ por 13 minutos.

Finalmente, los meristemos fueron teñidos por 2 horas en una solución de Feulgen con 5 gotas de celulosa-pectinasa al 5\%. Se hizo un aplastamiento al meristemo y se obtuvieron así las preparaciones para la observación de los cromosomas mitóticos. Las la- 
TABLA 1. Colector, número de colecta y localidad de los ejemplares de Nyctocereus estudiados.

\begin{tabular}{lll}
\hline Especies & Colector & Localidad \\
\hline & Sheinvar 3390 & Tezontepec, Hgo. \\
& Sheinvar 1072 & Tepalcatepec, Hgo. \\
Nyctocereus castellanosii & Sheinvar 2580 & Cerro Sta. Isabel, Pachuca, Hgo. \\
& Sheinvar 2202 & Zapotlán, Hgo. \\
& Sheinvar 2258 & Ixtapa-Zihuatanejo, Gro. \\
Nyctocereus & Sheinvar 3272 & La Piedad, Mich. \\
serpentinus & Sheinvar 2177 & Cerro Gordo, Pachuca, Hgo. \\
Nyctocereus serpentinus & Sheinvar 3000 & Temascalapa, Méx. \\
var splendens & Sheinvar 3442 & Temascalapa, Méx. \\
& &
\end{tabular}

minillas se hicieron permanentes por el método de hielo seco (Conger y Fairchild, 1953) y montadas en bálsamo de Canadá.

Para el análisis de la estructura de los cromosomas se observaron al menos 20 células provenientes de dos o tres individuos de cada colecta y eran corroborados con los cromosomas obtenidos de las plántulas germinadas a partir de semillas de los individuos adultos. El cariotipo se elaboró con las tres mejores metafases. Las fotografías se tomaron usando un fotomicroscopio III Zeiss y se dibujaron usando una cámara lúcida Zeiss. Se clasificaron de acuerdo con Levan et al. (1964). Se obtuvo así el número fundamental del cariotipo (n.f.) que corresponde al número de brazos de los cromosomas de acuerdo a Matthey (1945), el índice de asimetría (T.F.\%) para cada cariotipo según Sinha y Roy (1979) y la longitud total de la cromatina.

\section{Resultados}

El cariotipo para las dos especies y una variedad es: Nyctocereus castellanosii Scheinvar. $2 n=22$ (fig. 1B, $2 \mathrm{~A}$, tabla 2). Los cromosomas variaron de $1.85-2.89 \mu \mathrm{m}$ de longitud. Todos fueron metacéntricos, tres pares de ellos con constricción secundaria, su n.f. $=2$. La lontitud total de la cromatina es de $25.16 \mu \mathrm{m}$ y su índice de asimetría T.F. $\%=40.07$.

Nyctocereus serpentinus (Lag. y Rod.) Britt. y Rose. $2 n=22$ (figs. 1C, $2 \mathrm{~B}$ y tabla 2). Los cromosomas variaron de 1.57-3.22 $\mu \mathrm{m}$ de longitud. Todos fueron metacéntricos, tres pares con constricción secundaria; su n.f. $=22$. La longitud total de la cromatina es de $23.53 \mu \mathrm{m}$ y su índice de asimetría T.F.\% $=40.08$

N. serpentinus var. splendens (DC) Berg. $2 n=22$ (figs. 1A, 2C y tabla 2). Los cromosomas variaron de $1.62-2.56 \mu \mathrm{m}$ de longitud. Presentó nueve pares de cromosomas metacéntricos y dos parés de submetacéntricos; estos últimos presentaron doble constricción así como un par de los metacéntricos. Su n.f. $=22$. La longitud total de la cromatina es de $22.11 \mu \mathrm{m}$ y su índice de asimetría T.F. $\%=38.67$. 
TABLA 2. Análisis cariotípico de dos especies y una variedad de Nyctocereus.

\begin{tabular}{|c|c|c|c|c|c|c|c|}
\hline Especies & $2 n$ & $\begin{array}{c}\text { Intervalo } \\
\text { de longitud } \\
\text { de los cro- } \\
\text { mosomas } \\
(\mu \mathrm{m})\end{array}$ & $\begin{array}{l}\text { Fórmula } \\
\text { cariotípica }\end{array}$ & $n f$ & $\begin{array}{l}\text { Construccio- } \\
\text { nes secun- } \\
\text { darias }\end{array}$ & $\begin{array}{l}\text { Longitud } \\
\text { total de la } \\
\text { cromati- } \\
n a \pm E E \\
(\mu \mathrm{m})\end{array}$ & $\begin{array}{c}\text { Indice de } \\
\text { asime- } \\
\text { tría T.F. } \\
\text { (\%) }\end{array}$ \\
\hline N. castellanosii & 22 & $1.85-2.89$ & $11 \mathrm{~m}$ & 22 & $3 \mathrm{~m} \mathrm{Cs}$ & $25.16 \pm 0.91$ & 40.07 \\
\hline N. serpentinus & 22 & $1.57-3.22$ & $11 \mathrm{~m}$ & 22 & $3 \mathrm{~m} \mathrm{Cs}$ & $23.53 \pm 0.83$ & 40.08 \\
\hline $\begin{array}{l}\text { N. serpentinus var. } \\
\text { splendens }\end{array}$ & 22 & $1.62-2.56$ & $9 m+2 s m$ & 22 & $1 \mathrm{~m} \mathrm{Cs} ; 2 \mathrm{smCs}$ & $22.11 \pm 0.56$ & 38.67 \\
\hline
\end{tabular}

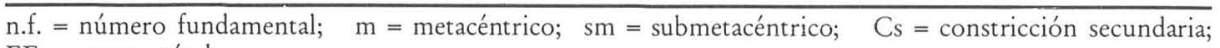
$\mathrm{EE}=$ error estándar.

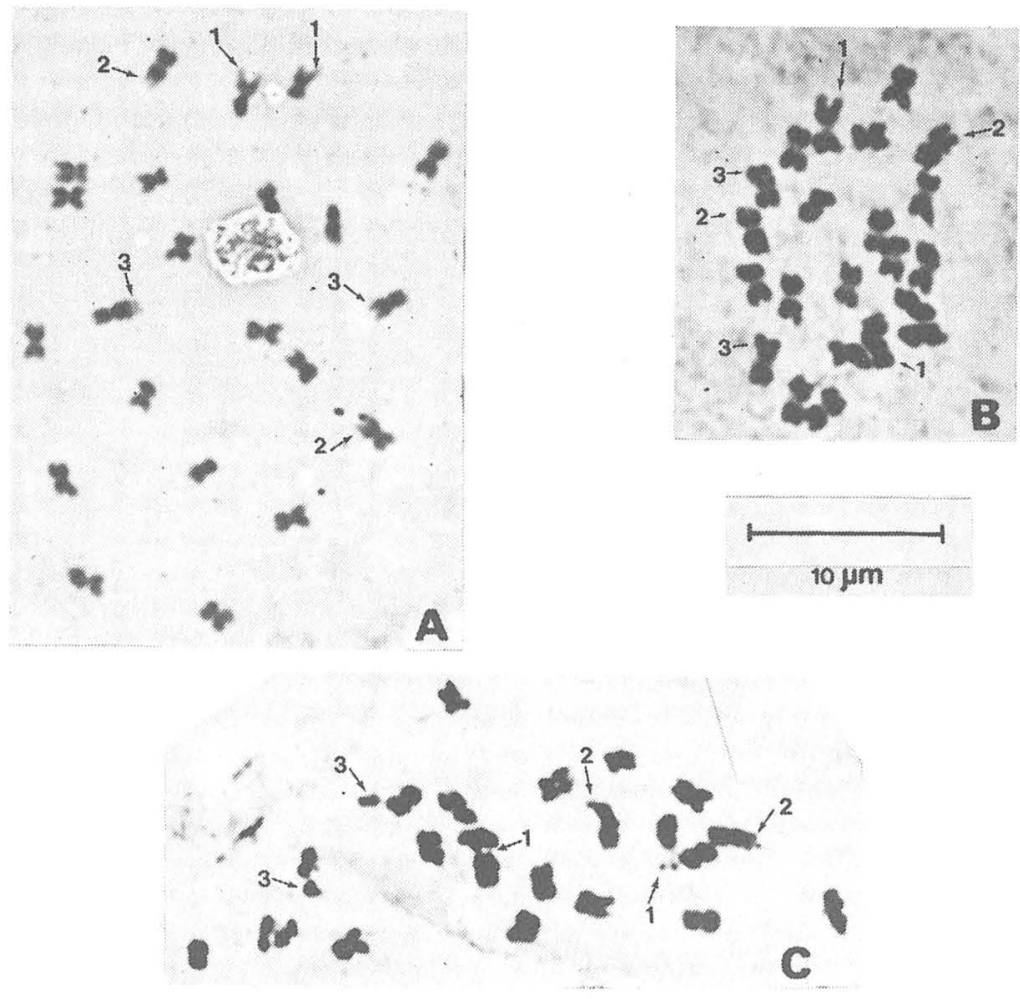

Fig. 1. Cromosomas de: A. Nyctocereus castellanosii, $2 n=22 ;$ B. N. serpentinus, $2 n=22$; C. $N$. serpentinus var. splendens, $2 n=22$. Las flechas y los números muestran los cromosomas con satélite. 


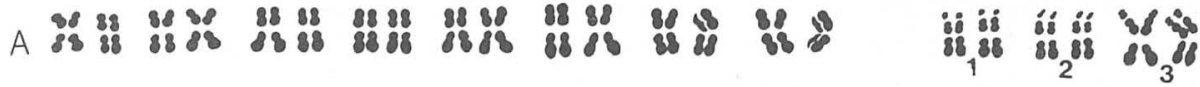

N. castellanosii

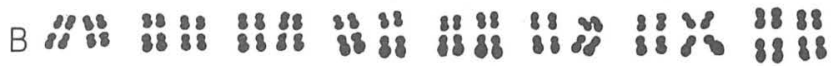

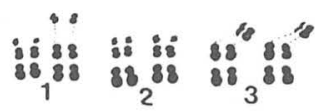

N. serpentinus

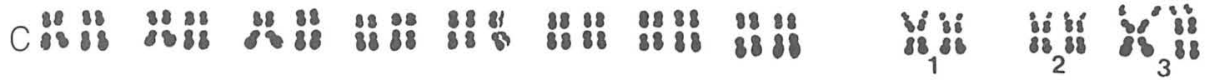 \\ N. serpentinus var. splendens

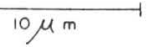

Fig. 2. Cariotipo de: A. Nyctocereus castellanosii; B. N. serpentinus; C. N. serpentinus var splendens. Los números indican los cromosomas con satélite.

La longitud total de la cromatina de los tres cariotipos se comparó por medio de una prueba de $t$ de Student para comparación de medias. La longitud total de la cromatina de $N$. castellanosii de $25.16 \mu \mathrm{m}$ resultó significativamente diferente a $5 \%$ a la de $N$. serpentinus con un valor de $23.53 \mu \mathrm{m}$ y a la de $N$. serpentinus var. splendens de $22.11 \mu \mathrm{m}$. En tanto que la primera y la última resultaron significativamente distintas al 1 por ciento.

\section{DiSCUSIÓN Y CONCLUSIONES}

El número cromosómico somático para las especies de Nyctocereus castellanosii y $N$. serpentinus y la variedad $N$. serpentinus var. splendens fue $2 n=22$, congruente con lo reportado para $N$. serpentinus por Beard (1937). Las dos especies y la variedad estudiadas fueron diploides. Podría por esto considerarse al género como citológicamente homogéneo; este punto de vista es acorde con los resultados obtenidos para otros géneros de la familia (Spencer, 1955; Pinkava y Mc Leod, 1971; Hiroshi et al., 1976; Weedin y Powell, 1978; Johnson, 1978 y 1980).

Sin embargo, no se observaron poliploidías como sucede en otras cactáceas del género Mammillaria donde Johnson (1978 y 1980) reporta especies tetraploides y hexaploides y al analizar la causa encuentra anormalidades en la meiosis cuando sucede la formación de las tétradas. Remski (1954) también reporta tetraploides, hexaploides y 24 ploides en especies de este género.

Nyctocereus castellanosii y $N$. serpentinus presentaron solamente cromosomas metacéntricos y $N$. serpentinus var. splendens, presentó dos pares de submetacéntricos que podrían ser el resultado de inversiones pericéntricas y de translocaciones desiguales de porciones de brazos de los cromosomas (Stebbins, 1971) o por pérdida de segmentos 
heterocromáticos durante el proceso de evolución y por deleciones que ocurrieron (Sihna y Roy, 1979). Este hecho se refleja también al considerar la longitud total de la cromatina donde $N$. castellanosii resultó tener el mayor valor con $25.16 \mu \mathrm{m}$, siguiéndole $N$. serpentinus con un valor intermedio de $23.53 \mu \mathrm{m}$ y $N$. serpentinus var. splendens con $22.11 \mu \mathrm{m}$.

En virtud de estas comparaciones del cariotipo, pudieron considerarse diferentes los tres taxa analizados. $N$. castellanosii y $N$. serpentinus resultaron con cariotipos similares en tanto que $N$. serpentinus var. splendens presentó dos pares de cromosomas submetacéntricas y la menor longitud de la cromatina, lo que pudiera indicar que es el cariotipo que más cambios ha sufrido a través del tiempo.

\section{LITERATURA CITADA}

BEARD, E.C. 1937. Some chromosome complements in the Cactaceae and a study of meiosis in Echinocereus papillosus. Bot. Gaz. 99:1-21.

Bravo-Hollis, H. 1978. Las cactáceas de México. 2a. ed., Universidad Nacional Autónoma de México, México, Vol. 1.

COnger, A.D. y L.M. FairChILD. 1953. A quick freeze method for making smear slides permanent. Stain Technology. 28:281-283.

Green, M.D., J.P. BOGART y E.H. ANTONY. 1980. An interactive, microcomputer based karyotype analysis system for phylogenetic cytotaxonomy. Compt. Biol. Med. 10:219-227.

Hiroshi, I., SHimizu y N. Kondo. 1976. Chromosome numbers and their bearing on the geographic distribution in the subfamily Opuntoideae. (Cactaceae). Rep. Inst. for Breed. Res. Tokyo Univ. Agric. 4:1-10.

Johnson, M.A.T. 1978. Diploid cytotypes in Mammillaria prolifera and three other Mammillaria species. Cact. Succ. J. Gt. Brit. 40(1):9-12.

- 1980. Further cytological investigations in Mammillaria prolifera and other Mammillaria species. Cact. Succ. J. Gt. Brit. 42(2):43-47.

Levan, A., K. Fredga y A.A. Sandberg. 1964. Nomenclature for centromeric position on chromosome. Hereditas 52:201-220.

Matthey, R. 1945. L'evolution de la formule chromosomiale chez las vertébrés. Experientia 1:50-78.

PINKAVA, D.F. y M.G. MCLEOD. 1971. Chromosome numbers in some cacti of Western North America. Brittonia 23:171-176.

RemSKI, M.F. 1954. Cytological investigations in Mammillaria and some associated genera. Bot. Gaz. 116:163-171.

Scheinvar, L. 1982. Las cactáceas del Valle de México. Tesis doctoral, Facultad de Ciencias, Unam, México, $724 \mathrm{pp}$.

SinHA, SS. y H. RoY. 1979. Cytological studies in the genus Phaseolus I. Mitotic analysis in fourteen species. Cytologia 44:191-199.

Spencer, J.L. 1955 A cytological study of the Cactaceae of Puerto Rico Bot. Gaz. 117:33-37.

STEBbins, G.L. 1971. Chromosome evolution in higher plants. Edward Arnold Publ. Ltd, Londres.

STOCK WELl, P. 1935. Chromosome numbers of some of the Cactaceae. Bot. Gaz. 96:565-570.

Weedin, J. y A.M. Powell. 1978. Chromosome numbers in Chihuahua Desert Cactaceae. Trans-Pecos, Texas. Amer. J. Bot 65(5):531-537. 